\title{
Flash-induced enhancements in the proton NMR relaxation rate of Photosystem II particles
}

\author{
A.N. Srinivasan and R.R. Sharp \\ Department of Chemistry, The University of Michigan, Ann Arbor, MI 48109 (U.S.A.)
}

(Received December 17th, 1985)

Key words: Photosynthetic water oxidation; Manganese; Oxygen-evolving complex; NMR; S state; Proton relaxation enhancement

Flash-induced enhancements in the NMR spin-lattice relaxation rate of solvent protons have been detected in suspensions of Photosystem II particles. The relaxation enhancements are small (less than $1 \%$ of background) and have been detected using signal-averaging techniques. The enhancements correlate with the known properties of the $S$ states with respect to (1) decay kinetics, (2) extractants of manganese, (3) sensitivity to atrazine-type inhibition of electron transport, (4) reagents which accelerate S-state decay (ADRY reagents), and (5) the two-flash retardation in the S-state advancement that is produced by low concentrations of $\mathrm{NH}_{2} \mathrm{OH}$. The transient proton relaxation enhancement observed after a single flash arises from a strongly relaxing paramagnetic species that is produced by the $S_{1} \rightarrow S_{2}$ transition of the water-oxidizing center. The appearance of such a species on an oxidative transition is suggestive of an $\mathrm{Mn}$ (III) $\rightarrow$ Mn(IV) oxidation.

\section{Introduction}

The photosynthetic oxidation of water to molecular oxygen takes place on the inner surface of the chloroplast thylakoid membrane, where the reaction is mediated by a catalytic center thought to contain four manganese ions [1-5]. The catalytic cycle of water oxidation involves a sequence of five oxidation states, called the $S$ states [6,7], which couple the one-electron photooxidation reactions at the Photosystem II trap to the four-electron oxidation of water. Evidence has been presented supporting the participation of manganese redox chemistry in the S-state transitions [8-14],

\footnotetext{
Abbreviations: Mes, 4-morpholineethanesulfonic acid; Chl, chlorophyll; PS, Photosystem; DCMU, 3-(3,4-dichlorophenyl)1,1-dimethylurea; CCCP, carbonyl cyanide $m$-chlorophenylhydrazone; ADRY, acceleration of the deactivation reactions of the water-oxidizing enzyme system.
}

but relatively little structural or electronic information on the metal center is available.

Within the past few years, both ESR and optical signatures of the $S$ states have been reported. Low-temperature ESR spectroscopy has revealed a 17-20 line signal centered near $g=2.0$ that is associated with the $S_{2}$ state in membranes that have been dark-adapted for a period of several hours [9-11,15-18]. A broad $g=4.1$ signal which also apparently arises from the S-state system has been observed upon illumination of PS II particles [15]. Period-4 optical transients associated with S-state transitions have also been detected in the ultraviolet [13,19-22] and the near infrared [14].

NMR relaxation of solvent protons has also been exploited as a probe of membrane-bound manganese in the photosynthetic system. Membrane-bound manganese in an electronic orbital singlet state provides a highly efficient relaxation trap for the magnetisation of solvent protons. 
Wydrzynski, Govindjee and their co-workers $[23,24]$ have reported flash-induced changes of the proton spin-spin relaxation rate $R_{2}$ of thylakoid suspensions. A single flash was found to produce an $R_{2}$ decrease with a magnitude of the order of 2 $\mathrm{s}^{-1}$, which was attributed to a $\mathrm{Mn}(\mathrm{II}) \rightarrow \mathrm{Mn}$ (III) oxidation on the $S_{1} \rightarrow S_{2}$ transition [25]. Attempts to repeat these experiments by the Illinois group $[26,27]$ or by workers in this laboratory [28] have not been successful, however. The latter investigations indicated that the observed $T_{2}$ changes may have been caused by oxidation of adventitious $\mathrm{Mn}$ (II) by superoxide produced on the reducing side of PS I [29-32]. When EDTA is present in the suspending medium and when superoxide formation is suppressed by addition of $\mathrm{Fe}(\mathrm{CN})_{6}^{-3}$, the flash-induced $R_{1}$ and $R_{2}$ changes were found in disappear at the $\pm 1 \%$ level of measurement precision [28].

This communication reports an extension of our previous proton relaxation enhancement studies using improved instrumentation for which the measurement precision is at the $\pm 0.1 \%$ level, i.e., one order of magnitude higher than in previous studies. Substantially enhanced signal/noise ratios have been achieved through the use of signal averaging of experimental data as well as through improvements in the noise figure of the detection system. These measurements have revealed the presence of small (less than 1\%) proton relaxation enhancement transients following flash illumination of suspensions of PS II particles. The transients decay on a time-scale $\left(t_{1 / 2}=20-50 \mathrm{~s}\right)$ that is suggestive of the $S_{2}$ and $S_{3}$ states. S-state involvement has been confirmed by the sensitivity of the phenomenon to known extractants of manganese in PS II, to DCMU (an atrazine-type inhibitor of electron transport), to agents which accelerate S-state decay, and to non-extracting concentrations of $\mathrm{NH}_{2} \mathrm{OH}$, which retard the $\mathrm{S}$ state flash cycle.

These NMR experiments demonstrate the formation of a strongly relaxing center upon advancement of the $S_{1}$ state. Such centers are characteristic of paramagnetic metal ions with orbitally nondegenerate electronic ground states. Among the common manganese oxidation states, these include only high-spin octahedral $\mathrm{Mn}$ (II) and $\mathrm{Mn}(\mathrm{IV})$. The formation of a strongly relaxing center following a single flash suggests that the $\mathrm{S}_{1} \rightarrow \mathrm{S}_{2}$ transition involves an oxidation of $\mathrm{Mn}(\mathrm{III})$ to $\mathrm{Mn}(\mathrm{IV})$.

\section{Materials and Methods}

Membranes enriched in Photosystem II were prepared by the method of Berthold et al. [33], as modified by Ghanotakis et al. [34], divided into $0.5 \mathrm{ml}$ aliquots and stored at $190 \mathrm{~K}$ in a buffer containing $400 \mathrm{mM}$ sucrose $/ 15 \mathrm{mM} \mathrm{NaCl} / 40 \mathrm{mM}$ $\mathrm{Na} / \mathrm{Mes}$ ( $\mathrm{pH}$ 6.0). Control rates of oxygen-evolution activity were typically $450 \mu \mathrm{mol} \mathrm{O}_{2}$ per mg $\mathrm{Chl}$ per ml. Samples were thawed in total darkness and then dark adapted on ice for at least $3 \mathrm{~h}$ prior to measurement. This prolonged dark adaption insured relaxation from active to resting states of the manganese center as reported by Beck et al. [15]. Care was taken to keep samples in complete darkness from the time of thawing to the completion of the experiment except during actinic flashes. After dark adaptation, $0.5 \mathrm{mM}$ EDTA and $1.5 \mathrm{mM} \mathrm{K}_{3} \mathrm{Fe}(\mathrm{CN})_{6}$ were added to the stock suspension. Tris extraction was conducted by pelleting a stock suspension of PS II membranes, resuspending the membranes in $0.8 \mathrm{M}$ Tris buffer (pH 8), and incubating for $1 \mathrm{~h}$ in room light. The extracted sample was then pelleted and resuspended in the storage buffer (see above). Extracting or non-extracting concentrations of $\mathrm{NH}_{2} \mathrm{OH}$ ( $2 \mathrm{mM}$ or $50 \mu \mathrm{M}$ as indicated) were added to the sample in the presence of EDTA at least $15 \mathrm{~min}$ before the start of measurements. NMR samples comprised $30 \mu 1$ of the PS II suspension contained in a circular flat cell $(8.0 \times 0.5 \mathrm{~mm})$ of a design similar to that described previously [35].

Flashes were obtained from a high-pressure xenon source (Xenon Corporation, Model 457 power supply with a Novatron 722 flash lamp). Flashes had a half width of $3 \mu \mathrm{s}$ and a dissipated electrical energy of 15-25 J per pulse. The radiant output was focused with a parabolic mirror and a condensing lens into a lucite light pipe by means of a right-angle prism. The sample cell, which was milled into the end of the light pipe, was positioned in the receiver coil of the NMR spectrometer.

NMR relaxation measurements were conducted at $20.7 \mathrm{MHz}, 21-22^{\circ} \mathrm{C}$, using the phase-shifted 
triplet sequence and a pulsed NMR spectrometer described previously $[36,37]$. Improvements in the instrument sensitivity were made by winding a receiver coil with improved filling factor for the flash cell, and by the addition of new signal preamplifiers with an improved noise figure. In addition, software was written to provide more efficient data acquisition and analysis and to permit the averaging of data from different experimental runs. With these improvements, the measurement precision of data averaged over 25 runs was typically $\pm 0.1 \%$ with a $30 \mu 1$ sample, which is approx. one order of magnitude better than that of previously reported data from this laboratory.

Prior to the measurement sequence, individual samples were permitted to temperature equilibrate in the probe for $10 \mathrm{~min}$, given one pre-flash, and then incubated for $6 \mathrm{~min}$ in the NMR probe in order to set the $S_{1}$ state prior to the start of each measurement sequence. Each measurement sequence comprised fifty $R_{1}$ determinations at 10-s intervals. The first 12 and the last 32 determinations were averaged over two points. The plotted data in Figs. 1-3 are therefore shown as 28 measurements; the first 6 and the last 16 at $20-\mathrm{s}$ intervals are 2-point averages, while the 7 th-12th points at 10-s intervals are unaveraged. The flash spacing in multiple flash trains was $600 \mathrm{~ms}$. Each individual sample was used for four separate measurement sequences $\left(51.7 \mathrm{~min}\right.$ at $\left.21^{\circ} \mathrm{C}\right)$ before replacement, except for the experiments of Fig. 3, where each sample was used for only two measurement sequences. Control experiments in this and other laboratories $[15,38]$ indicate that the oxygen-evolution activity of PS II preparations is stable over this time-scale. A very slow drift in the dark $R_{1}$ value usually occurred due to slow temperature equilibration of the lucite light pipe. This was corrected by subtracting a linear baseline defined by the average $R_{1}$ of the pre-flash periods of successive measurement sequences.

\section{Results}

Figs. 1-3 summarize the results of measurements of the enhancements produced by actinic flashes in the NMR spin-lattice relaxation rate of solvent protons in suspensions of PS II-enriched membranes. $R_{1}$ measurements were initiated 150

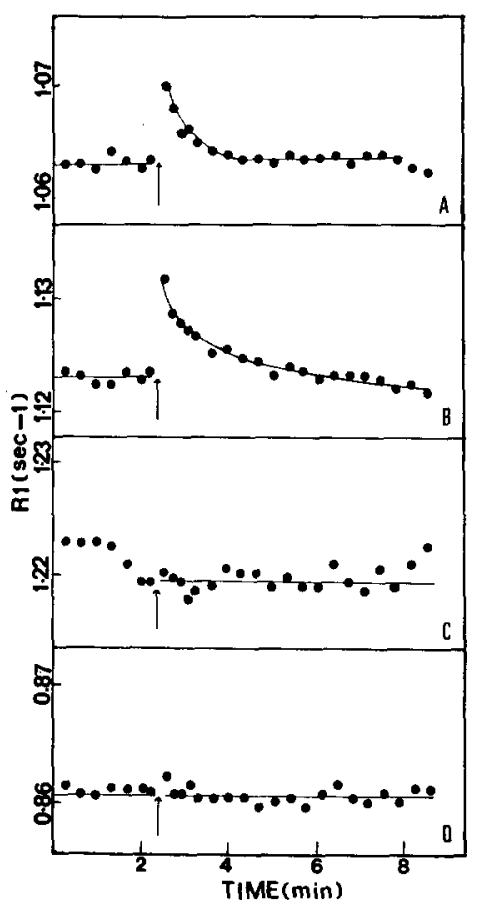

Fig. 1. Proton solvent relaxation rate as a function of time following flash illumination of suspensions of PS II particles. Relaxation transient following a single flash (A) or two flashes (B) applied to control suspensions. Also shown are effects of one flash applied to membranes extracted with $\mathrm{NH}_{2} \mathrm{OH}$ / EDTA (C) and $0.8 \mathrm{M}$ Tris buffer (D) as described under Methods. NMR samples contained $1.5 \mathrm{mM} \mathrm{Fe}(\mathrm{CN})_{6}^{-3}$ and 0.5 mM EDTA. The Tris-extracted sample contained $350 \mu \mathrm{M}$ ascorbate. Chlorophyll concentration was $2.2-2.4 \mathrm{mg} \cdot \mathrm{ml}^{-1}$. Each trace is an average of 24 experiments.

ms after the final flash; determination of a single $R_{1}$ value required approx. $2 \mathrm{~s}$ in order to define the spin-lattice decay. Thus the reported $R_{1}$ values should be viewed as an average over a 2-s interval beginning at the indicated time. The effect of a single flash on the $R_{1}$ of a PS II suspension is shown in Fig. 1a. $R_{1}$ increased abruptly by about $0.007 \mathrm{~s}^{-1}$, and subsequently decayed to the baseline with a half-time of $25 \mathrm{~s}$. The result of a two flash experiment is shown in Fig. 1b. The initial $R_{1}$ increase was somewhat larger $\left(0.009 \mathrm{~s}^{-1}\right)$, and the decay was slower $\left(t_{1 / 2} \approx 40-50 \mathrm{~s}\right)$. The flash illumination was not fully saturating, however; and thus further work is needed to characterize the $S_{2} \rightarrow S_{3}$ transition.

The observed decay kinetics of these enhancements suggested involvement of the $\mathrm{S}$ states. To 


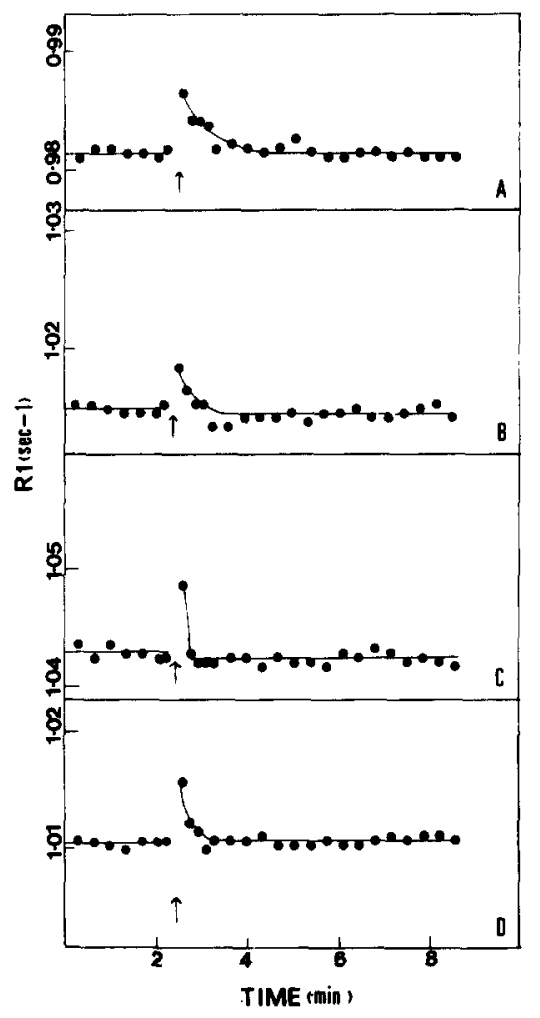

Fig. 2. Flash-induced relaxation transients in PS II suspensions in the presence of the ADRY reagent $\mathrm{CCCP}(\mathrm{A}-\mathrm{C})$ and the atrazine-type electron-transport inhibitor DCMU (D). Experimental conditions are as described in the legend of Fig. 1, except for the inclusion of $0.1 \mu \mathrm{M}$ (A), $0.25 \mu \mathrm{M}$ (B), or $0.5 \mu \mathrm{M}$ (C) CCCP, or $10 \mu \mathrm{M}$ DCMU (D) in the suspension medium. Each trace is an average of 25 experiments.

test this possibility, we recorded the effects of actinic flashes on suspensions that had been exposed to extracting concentrations of $\mathrm{NH}_{2} \mathrm{OH}$ (Fig. 1c) and Tris buffer (Fig. 1d). Both extraction techniques effectively suppressed the flash-induced proton NMR relaxation enhancements.

As a further control, we studied the effects of DCMU, an atrazine-type inhibitor of electron transport, on the flash-induced $R_{1}$ enhancements (Fig. 2d). DCMU blocks electron transport between $Q_{A}$ and $Q_{B}$ on the reducing side of PS II [39]. In the presence of DCMU, a single charge separation is possible in PS II, leading to the formation of the $S_{2} \cdot Q_{A}^{-}$state. Charge recombination in the $S_{2} \cdot Q_{A}^{-}$state is accelerated relative to $S_{2} \cdot Q_{B}^{-}$due to the lower potential of the reduced acceptor in the former $[40,41]$, leading to more

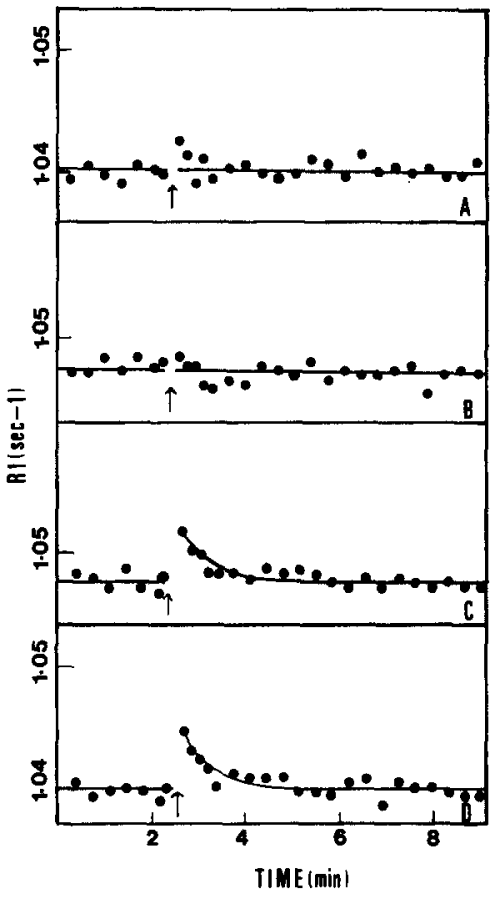

Fig. 3. Effect of a non-extracting concentration of $\mathrm{NH}_{2} \mathrm{OH}$ on the flash-induced proton relaxation enhancement in suspensions of PS II particles. Experimental conditions are as described in Fig. 1 except for the inclusion of $50 \mu \mathrm{M} \mathrm{NH}{ }_{2} \mathrm{OH}$ Figs. 3A-D show the relaxation transient following 1, 2, 3 and 4 flashes, respectively. Traces $A$ and $D$ are averages of 12 experiments, traces $B$ and $C$ are averages of 16 experiments.

rapid decay kinetics of the $S_{2}$ state. The observed flash-induced $R_{1}$ enhancement is consistent with these properties. The flash-induced increase in $R_{1}$ reflects the $S_{1} \rightarrow S_{2}$ transition, and the decay kinetics of the $S_{2}$ state are accelerated (compare Fig. 1a and Fig. 2d).

CCCP is a reagent which, at submicromolar concentrations, accelerates the deactivation reactions of $S_{2}$ and $S_{3}$ (ADRY reagent, $[42,43]$ ). The effects of 100-500 nM CCCP on the flash-induced proton NMR relaxation enhancement is. shown in Figs. 2a-c. An initial $R_{1}$ enhancement persists throughout this concentration range, but its decay kinetics are markedly accelerated at $500 \mathrm{~nm}$. The threshold of this effect occurs near $100 \mathrm{nM}$, which is consistent with the behavior of CCCP in oxygen flash yield studies [44].

As a final control, we recorded the effects of non-extracting concentrations of $\mathrm{NH}_{2} \mathrm{OH}$. In the presence of $50 \mu \mathrm{M} \mathrm{NH} \mathrm{NH}_{2} \mathrm{OH}$, the normal cycle of 
oxygen-flash yields is retarded by two flashes, with the maximum flash yield occurring on the fifth flash rather than on the third. This behavior has been explained previously in terms of the binding and subsequent flash oxidation of $\mathrm{NH}_{2} \mathrm{OH}$ [45], or alternatively, as the reduction of the $S$ states to a non-physiological level, $S_{-1}[46,47]$. In either model, the $S_{1} \rightarrow S_{2}$ transition is delayed until the third flash. Fig. 3 shows the effect of 50 $\mu \mathrm{M} \mathrm{NH} \mathrm{NH}_{2} \mathrm{OH}$ on the flash-induced proton NMR relaxation enhancement of PS II suspensions. A two-flash delay in the appearance of the proton NMR relaxation enhancement was observed, confirming the involvement of the $S$ states.

\section{Discussion}

The experiments described here were undertaken to investigate the possible involvement of paramagnetic centers associated with S-state formation as relaxation traps for the magnetisation of solvent protons. Earlier measurements from this laboratory failed to detect flash-induced relaxation enhancements in suspensions of thylakoids or of PS II particles [28]. In the present work, we have extended the level of precision by approx. one order of magnitude over that of the previous study through the combined use of signal averaging and by improvements in the instrumental sensitivity. At the current level of precision, $R_{1}$ changes as low as $0.002 \mathrm{~s}^{-1}$ are detectable. We report here the detection of flash-induced $R_{1}$ enhancements with magnitudes in the range $0.007-0.009 \mathrm{~s}^{-1}$ in suspensions of PS II particles.

The behavior of the relaxation transients indicates an origin associated with paramagnetic centers that comprise the S states of our PS II-enriched membrane preparations. Evidence supporting this conclusion includes the following.

(1) The decay kinetics of the proton NMR relaxation enhancement coincides with the reported behavior of $S_{1}$ and $S_{2}$ in PS II preparations $[38,48,49]$.

(2) Treatments which abolish oxygen-evolution activity through selective extraction of manganese from the membrane (incubation with $2 \mathrm{mM}$ $\mathrm{NH}_{2} \mathrm{OH}$ or alkaline Tris/EDTA) were found to suppress the relaxation enhancements.

(3) DCMU, an atrazine-type inhibitor of electron transport, permits a single S-state transition in PS
II and, after a single flash, promotes accelerated decay of $S_{2}$ through charge recombination with the electron on the reduced acceptor, $\mathrm{Q}_{\mathrm{A}}^{-}$. Membranes incubated with DCMU exhibit a proton NMR relaxation enhancement after one flash; the enhancement decays with kinetics that are accelerated with respect to control values.

(4) CCCP, an ADRY reagent which accelerates the decay of $S_{2}$ and $S_{3}$ in oxygen-flash-yield experiments, likewise accelerates the decay of the proton NMR relaxation enhancement after a single flash. The concentration dependence of this effect is similar to that previously reported in oxygenflash-yield studies.

(5) Non-extracting concentrations of $\mathrm{NH}_{2} \mathrm{OH}$, which have previously been shown to retard the oxygen-flash cycle by two flashes, also retard the appearance of the flash-induced relaxation transient from the first to the third flash.

These observations clearly indicate that the observed flash-induced relaxation transients arise from paramagnetic centers associated with S-state formation. However, several aspects of these phenomena are unclear at present. One of these is the mechanism of magnetic coupling between the paramagnetic center and the pool of solvent protons. The usual interpretation of proton NMR relaxation enhancement effects in solutions of metalloproteins involves the transfer of spin magnetisation from the bulk solvent into the inner sphere of the paramagnetic metal ion by chemical exchange effects, followed by efficient paramagnetic relaxation of inner sphere protons by the metal ion [50-52]. Outer sphere relaxation mediated by direct dipole couplings may also be significant when chemical exchange is slow [52].

It is interesting in the present context to note that a third relaxation mechanism may also be important for membrane-bound paramagnetic centers which are sequestered from the aqueous phase. This mechanism follows a dipolar relaxation pathway from the solvent into the membrane phase. The nuclear spin system of the membrane is very tightly coupled by internal dipolar interactions, and proton magnetisation can travel by relatively efficient spin diffusion processes to paramagnetic centers, where the spin energy is thermalized. The spin-diffusion mechanism does not require that paramagnetic centers be accessi- 
ble to the solvent; furthermore, the lipid phase, rather than the inhibiting magnetisation transfer, may act as a chemical amplifier. The actual mechanism of the flash-induced proton NMR relaxation enhancement transients reported here is not yet clear, but it is worth noting that in general the underlying relaxation processes can occur over a very long range if mediated by spin diffusion; proton NMR relaxation enhancement effects require neither direct magnetic coupling to the solvent nor rapid solvent exchange with the inner coordination sphere of the paramagnetic center.

The chemical identity of the strongly relaxing species that is produced by a single flash is not yet certain, although $\mathrm{Mn}(\mathrm{IV})$ is a likely candidate. The main characteristic of an efficient nuclear relaxation trap is a long paramagnetic relaxation time and a high total spin. Species with long electronic relaxation times characteristically have orbitally nondegenerate ground states, where the zero-field splitting is small [53]. Among the manganese-oxidation states, $\mathrm{Mn}(\mathrm{II})$ and $\mathrm{Mn}(\mathrm{IV})$ in high-spin octahedral environments are candidates as strongly relaxing centers, while $\mathrm{Mn}(\mathrm{III})$ has very large static zero-field splittings and is very weakly relaxing. Thus the appearance of a strongly relaxing center upon the oxidation of the S-state system from $S_{1}$ to $S_{2}$ is suggestive of an $\mathrm{Mn}$ (III) $\rightarrow \mathrm{Mn}$ (IV) oxidation. The production of $\mathrm{Mn}$ (III) can be excluded due to the large zero-field splitting of the $\mathrm{d}^{4}$ configuration.

On the other hand, we cannot from the NMR data alone exclude the production of a spin- $\frac{1}{2}$ radical. However other spectroscopic data seem to indicate clearly that the $S_{1} \rightarrow S_{2}$ transition results in the formation of a mixed valence manganese cluster. The production of $S_{2}$ coincides with the appearance of the multiline ESR spectrum, which can be interpreted in terms of an odd spin state involving a spin-coupled dimer or tetramer of manganese $[10,11]$. In addition, the flash-induced infrared transients that have been related to the $S$ states are suggestive of an intervalence electronic transition of a mixed valence metal complex [14].

The specific oxidation state change that occurs on the $S_{1} \rightarrow S_{2}$ transition appears from the NMR data presented here to be $\mathrm{Mn}(\mathrm{III}) \rightarrow \mathrm{Mn}(\mathrm{IV})$. This conclusion is also suggested by the spectrum of the period-4 ultraviolet transient [13] as well as by the flash-induced shift to higher energy of the $\mathrm{X}$-ray $\mathrm{K} \alpha$ absorption edge of manganese [12] (although some caution may be warranted here, since the absorption edge position is very sensitive to changes in coordination geometry [54]). Taken together, the available data indicate that the $S_{1} \rightarrow$ $\mathrm{S}_{2}$ transition reflects the formation of a mixed valence state concomitant with the oxidation of $\mathrm{Mn}$ (III) to $\mathrm{Mn}$ (IV).

At present we are unable to quantitate proton NMR relaxation enhancements beyond the $S_{2}$ state due to the difficulty of obtaining saturating flashes. Such experiments are currently in progress.

\section{Acknowledgement}

Partial support of this research was obtained in the form of a grant from the United States Department of Agriculture Competitive Grants Research program (No. 82-CRCR-1-1047).

\section{References}

1 Cheniae, G.M. and Martin, I.F. (1970) Biochim. BIophys. Acta 197, 219-239

2 Cheniae, G.M. and Martin, I.F. (1971) Plant Physiol. 47, 568-575

3 Radmer, R. and Cheniae, G.M. (1976) in Topics in Photosynthesis (Barber, J., ed.), Vol. 2, pp. 303-368, Elsevier, Amsterdam

4 Chemiae, G.M. (1980) Methods Enzymol. 69, 349-363

5 Yocum, C.F., Yerkes, C.T., Blankenship, R.E., Sharp, R.R. and Babcock, G.T. (1981) Proc. Natl. Acad. Sci. USA 78, 7507-7511

6 Kok, B., Forbush, B. and McGloin, M. (1970) Photochem. Photobiol. 11, 457-475

7 Forbush, B., Kok, B. and McGloin, M. (1971) Photochem. Photobiol. 14, 307-321

8 Wydrzynski, T. and Sauer, K. (1980) Biochim. Biophys. Acta 589, 56-70

9 Dismukes, G.C. and Siderer, Y. (1980) FEBS Lett. 121 78-80

10 Dismukes, G.C. and Siderer, Y. (1981) Proc. Natl. Acad. Sci. USA, 78, 274-278

11 Dismukes, G.C., Ferris, K. and Watnick, P. (1982) Photochem. Photobiol. 3, 243-256

12 Goodin, D.B., Yachandra, R., Britt, R.D., Sauer, K. and Klein, M. (1984) Biochim. Biophys. Acta 767, 209-216

13 Dekker, J.P., Van Gorkom, H.J., Wensink, J. and Ouwehand, L. (1984) Biochim. Biophys. Acta 767, 1-9

14 Dismukes, C.G. and Mathis, P. (1984) FEBS Lett. 178, $51-54$

15 Beck, W.F., De Paula, J.C. and Brudvig, G.W. (1985) Biochemistry 24, 3043-3049 
16 Hansson, Ö. and Andreasson, L.-E. (1982) Biochim. Biophys. Acta 679, 261-268

17 Casey, J.L. and Sauer, K. (1984) Biochim. Biophys. Acta $767,21-28$

18 Zimmermann, J.L. and Rutherford, A.W. (1984) Biochim. Biophys. Acta 767, 160-167

19 Velthuys, B.R. (1981) in Proceedings of the 5th International Congress on Photosynthesis (Akoynoglou, G., ed.), Vol. 2, 75-85, Balaban International Science Services, Philadelphia, PA

20 Renger, G. and Weiss, W. (1983) Biochim. Biophys. Acta 722, 1-11

21 Dekker, J.P., Van Gorkom, H.J., Brok, M. and Ouwehand, L. (1984) Biochim. Biophys. Acta 764, 301-309

22 Dekker, J.P., Plijter, J.J., Ouwehand, L. and Van Gorkom, H.J. (1984) Biochim. Biophys. Acta 767, 176-179

23 Wydrzynski, T., Zumbulyadis, N., Schmidt, P.G. and Govindjee (1975) Biochim. Biophys. Acta 408, 349-354

24 Wydrzynski, T., Zumbulyadis, N., Schmidt, P.G., Gutowsky, H.S. and Govindjee (1976) Proc. Natl. Acad. Sci. USA 73, 1196-1198

25 Govindjee (1978) Natl. Acad. Sci. Lett. 1, 3-6

26 Govindjee and Wydrzynski, T. (1981) in Photosynthesis II. Electron Transport and Photophosphorylation (Akoyunoglou, G., ed.), pp. 293-303, Balaban International Science Service, Philadelphia, PA

27 Khanna, R., Rajan, S., Govindjee and Gutowsky, H.S. (1981) in Photosynthesis II. Electron Transport and Photophosphorylation (Akoyunoglou, G., ed.), pp. 307-316, Balaban International Science Services, Philadelphia, PA

28 Sharp, R.R. and Yocum, C.F. (1983) Photobiochem. Photobiophys. 5, 193-199

29 Robinson, H.H., Sharp, R.R. and Yocum, C.F. (1981) Arch. Biochem. Biophys. 207, 1-8

30 Robinson, H.H., Sharp, R.R. and Yocum, C.F. (1980) Biochem. Biophys. Res. Commun. 93, 755-761

31 Sharp, R.R. and Yocum, C.F. (1980) Biochim. Biophys. Acta 592, 185-195

32 Robinson, H.H., Sharp, R.R. and Yocum, C.F. (1980) Biochim. Biophys. Acta 593, 414-426

33 Berthold, D.A., Babcock, G.T. and Yocum, C.F. (1981) FEBS Lett. 134, 231-234
34 Ghanotakis, D.F., Babcock, G.T. and Yocum, C.F. (1984) FEBS Lett. 167, 127-130

35 Sharp, R.R. and Yocum, C.F. (1981) Biochim. Biophys. Acta 635, 90-104

36 Sharp, R.R. and Sen, R. (1982) Biophys. J. 40, 17-25

37 Haddy, A.H., Frasch, W.D. and Sharp, R.R. (1985) Biochemistry $24,7926-7930$

38 Seibert, M. and Lavorel, J. (1983) Biochim. Biophys. Acta 723, 160-168

39 Velthuys, B.R. and Amesz, J. (1974) Biochim. Biophys. Acta 333, 85-94

40 Bennoun, P. (1970) Biochim. Biophys. Acta 216, 357-363

41 Bouges-Bocquet, B., Bennoun, P. and Taboury, J. (1973) Biochim. Biophys. Acta 325, 247-254

42 Renger, G. (1969) Naturwissenschaften 56, 370

43 Renger, G. (1972) Biochim. Biophys. Acta 256, 428-439

44 Homann, P. (1971) Biochim. Biophys. Acta 245, 129-143

45 Bouges-Bocquet, B. (1973) Biochim. Biophys. Acta 292, $772-785$

46 Velthuys, B. and Kok, B. (1977) in Proceedings of the Fourth International Congress on Photosynthesis, pp. 397-405, Dr. W. Junk Publishers, Dordrecht, The Netherlands

47 Velthuys, B.R. and Visser, J.W.M. (1975) FEBS Lett. 55 , 109-112

48 Boussac, A., Maison-Peteri, B., Vernotte, C. and Etienne, A.-L. (1985) Biochim. Biophys. Acta 808, 225-230

49 Rutherford, A.W. and Inoue, Y. (1984) FEBS Lett. 165, $163-170$

50 Solomon, I. (1955) Phys. Rev. 99, 559

51 Bloembergen, N. and Morgan, L.O. (1961) J. Chem. Phys. $27,842-850$

52 Dwek, R.A. (1973) NMR in Biochemistry, Oxford University Press, Oxford, Ch. 9

53 Lewis, W.B. and Morgan, L.O. (1968) Paramagnetic Relaxation in Solutions in Transition Metal Chemistry (Carlin, R.L., ed.), Vol. 4, pp. 33-112, Marcel Dekker, New York

54 Smith, T.A., Penner-Hahn, J.E., Berding, M.A., Doniach, S. and Hodgson, K.O. (1985) J. Am. Chem. Soc. 107, 5945-5955 